\title{
A Reduced-Order Model based on the Coupled 1D-3D Finite Element Simulations for an Efficient Analysis of Hemodynamics Problems.
}

Eduardo Soudah, Riccardo Rossi, Sergio Idelsohn, Eugenio Oñate

\begin{abstract}
A reduced-order model for an efficient analysis of cardiovascular hemodynamics problems using multiscale approach is presented in this work. Starting from a patient-specific computational mesh obtained by medical imaging techniques, an analysis methodology based on a two-step automatic procedure is proposed. First a coupled 1D-3D Finite Element Simulation is performed and the results are used to adjust a reduced-order model of the 3D patient-specific area of interest. Then, this reduced-order model is coupled with the 1D model. In this way, threedimensional effects are accounted for in the 1D model in a cost effective manner, allowing fast computation under different scenarios. The methodology proposed is validated using a patient-specific aortic coarctation model under rest and non-rest conditions
\end{abstract}

Key words: Blood Flow, Boundary Conditions, Reduced-Order Models and Aortic coarctation

\section{Introduction}

The simulation of blood flow problems assumes a large importance in biomechanics due to the many potential fields of application. The use of realistic boundary conditions is essential to guarantee the performance and the accuracy of numerical simulations, especially in cardiovascular problems. In particular, the flow in arteries depends strongly on the outflow boundary conditions which model the downstream domain. The application of constant tractions as outlet boundary conditions

Eduardo Soudah, Riccardo Rossi, Sergio Idelsohn*, Eugenio Oñate.

International Center for Numerical Methods in Engineering (CIMNE). Technical University of Catalonia. Barcelona, 08034, Spain.

*Institució Catalana de Recerca i Estudis Avançats (ICREA)

Correspondence to: Eduardo Soudah, e-mail: esoudah@cimne.upc.edu 
for 3D domains represents the simplest possibility. Unfortunately, such conditions are not realistic and cause spurious pressure waves to become in the solution. Such waves travel along the artery network and distort the numerical solution. An efficient technique is thus needed to minimize these effects. Sophisticated outlet boundary conditions [14][15] aimed to minimizing such problem can be found in the literature. Others authors address the problem by applying geometrical multiscale modeling [1][2][7][18][19][24]. These approaches typically consist in the combination of models with different levels of approximation (3D, 1D and 0D models) each aimed at capturing particular features of the solution. 3D models are applied in regions where details of the local flow are needed. This is typically the case when the flow is strongly three dimensional or it tends to be turbulent. 1D models are typically used in the up-downstream domain of the 3D models, so that the whole arterial network can be described efficiently taking into account flow propagation effects. Zero dimensional models (or lumped models) are generally used to describe the lower level of the cardiovascular system or to model the heart. A typical problem that rises at the interface between the 1D and 3D domains is the mapping of the parabolic velocity distribution (assumed in the 1D model) to an "equivalent" distribution on the 3D inlet. Such mapping is not trivial since the discretized 3D model is generally not exactly circular. A proposal to solve the impasse can be found in [36]. In current work we opted for the simpler (but less accurate) option of applying a spatially uniform inlet velocity, with a total flow corresponding to the one of the 1D. This is only acceptable since our aim is not to compute the Wall Shear Stress (WSS) but rather to estimate the dissipation induced by the non-standard topology of the artery or area of interest. We also observe that the method we propose works under the assumption that the 3D inlet and outlet boundaries are approximately perpendicular to the centerline of the artery and positioned at points in which the flow can be reasonably approximated as 1D. It is interesting to remark that, as observed in the extensive review paper [16][22], the reliable computation of WSS, which is often the target of CFD simulations, depends on the availability of a sufficiently fine discretization of the boundary layer and is sensibly affected by the Fluid-Structure Interaction of the flow with the artery boundaries. The idea we leverage in the current work is that the extra dissipation induced by severe variations of the geometry is dominated by the appearance of turbulent effects within the volume. The evaluation of such effect requires a sufficiently fine discretization of the volume but does not put the extra requirements on the boundary layer mesh, and shall not be severely affected by the deformability of the walls, hence allowing the assumptions of considering the walls rigid which greatly simplifies the simulation and reduces the runtime. In [29] an extensive review of the most popular 1D models can be found. Recently, new models have been proposed [8][27][30] improving the viscoelastic behavior of the walls. However, the 1D models alone are not capable to capture in an effective way the energy losses due to the 3D geometrical shapes of the arteries, e.g, in stenotic arteries, aneurysms or other cardiovascular pathologies, such as aorta coarctation. Nevertheless, to discretize the whole 3D cardiovascular domain or coupled fluidstructure interaction (FSI) modeling is computational expensive and unfeasible in practical applications due to the numerical challenges involved. Despite its mod- 
eling shortcomings, geometrical multiscale models combined with patient-specific geometries remains the predominant approach for vascular blood flow. Such models allow quantifying the hemodynamics variables such as, flow reversal, flow separation and wall shear stress areas over the arterial wall in a non-invasive way useful for clinicians. Notable exceptions include the work of [14] [34] [31] on cerebral aneurysms.

In this paper, we propose a reduced-order Computational Fluid Dynamics(CFD) model specifically aimed to the estimation of the pressure gradient and the energy losses induced by stenosis in cardiovascular scenarios. The key features of our approach are: (1) a patient-specific anatomy and a computational mesh obtained during a routine clinical imaging session; (2) a coupled multiscale 0D-1D-3D model to approximate the energy losses induced by a narrowing of the artery lumen. The solution of the 3D model is used to train a reduced-order model which is aimed to capturing the pressure drop within two sections located upstream and downstream of the stenosis. Then the reduced-order model is integrated within the $1 \mathrm{D}$ model to create a $1 \mathrm{D}$ reduced-order model. Such model is able to simulate the energy losses and the flow distribution taking into account the patient-specific anatomy in real time under different scenarios. The ultimate goal is to validate the CFD framework with the energy losses through an aortic coarctation( $\mathrm{CoA})$ under resting and non-resting conditions of the patient. CoA of the aorta occurs approximately in $10 \%$ of patients with congenital heart defects and represents a narrowing of the descending aorta. Due to the reduction in the aorta descending diameter, high pressure gradients can appear across the CoA, resulting in an increased cardiac workload in the left ventricle during systole[10]. Investigation into the hemodynamics and bio-mechanical basis of the morbidity in CoA shows that the pressure gradient is dependent on the aorta area reduction, the flow rate and the physiological state of the patient: during non-rest conditions the pressure gradient can increase considerably and can provoke heart failure[11]. To measure the pressure gradient under these non-rest conditions, that are difficult to replicate in a clinic environment, is a biomedical challenge[4]. For is reason, a procedure that combines patient-specific image data and numerical tools to further understand the hemodynamics alterations, under resting and nonresting situations will allow clinicians to improve the diagnosis and define which should be the CoA treatment for the patient[10][13]. Some authors have used CFD models to study the hemodynamics in the CoA [11][20]. However, different numerical approaches might lead to different pressure predictions. The reduced-order methodology described in this paper has been implemented as part of the C++ finite element library KRATOS(www.cimne.com/kratos)[25]. KRATOS is a multiphysics simulation open source (LGPL licence) framework based on the stabilized Finite Element Method for analysis of the Navier-Stokes equations in viscous flows. Efficient and parallel solvers for 3-D fluid-structure interaction(FSI) [26] problems have been implemented in KRATOS that allow tackling large problems using supercomputers if available. 


\section{Computational Framework}

As previously stated, realistic boundary conditions are essential for simulating a appropriately behavior of the blood flow propagation. In this section we define a 1D FSI model coupled to a reduced-order model for cardiovascular analysis. The methodology used consists on a two step process. The first step (training phase) consists in estimating correctly the pressure drop between two sections of a 3D model by solving a geometrical multiscale problem. From the numerical point of view, we embed a 3D domain into a 1D network in order to perform 3D simulations within a consistent hemodynamics conditions provided by a 1D FSI model. A lumped model is used to simulate the peripheral vascular beds of the cardiovascular system. Once the first simulation is completed, the pressure drop predicted by the $3 \mathrm{D}$ model is used to train the $1 \mathrm{D}$ reduced-order model via a least square fitting procedure. The trained model is thus able to estimate the energy losses between the two areas selected in the 3D model. Once the reduced-order model is defined, a coupled 1D FSI-reduced-order model will be capable of estimating the patient-specific pressure drop under different rest or non-rest situations. The second step (computing phase) consists in setting different boundary conditions for the coupled 1D FSIreduced-order model to estimate the pressure drop (at any point) taking in account the 3D anatomical model. This will enable us to simulate different pathological situations taking into account the energy losses produced by the 3D model. Besides, this reduced-order approach brings down the computational costs significantly. The flow diagram of this scheme is shown in Fig.1.

\subsection{Mathematical model for the $1 D$ reduced-order model}

In this section we describe a non-linear 1D formulation and the reduced-order model proposed to account for the 3D effects caused by the patient-specific area of interest. In absence of branching, an artery may be considered as a cylindrical compliant tube which extends from $\mathrm{z}=0$ to $\mathrm{z}=\mathrm{L}$, where $\mathrm{L}$ is the the artery length. The artery takes into account the assumptions of axial symmetry, radial displacements, constant pressure on each section, no body forces and dominance of axial velocity. The governing system of equations for an incompressible newtonian fluid are derived by applying conservation of mass and momentum in a 1-D impermeable and deformable tubular control volume. These equations are: 


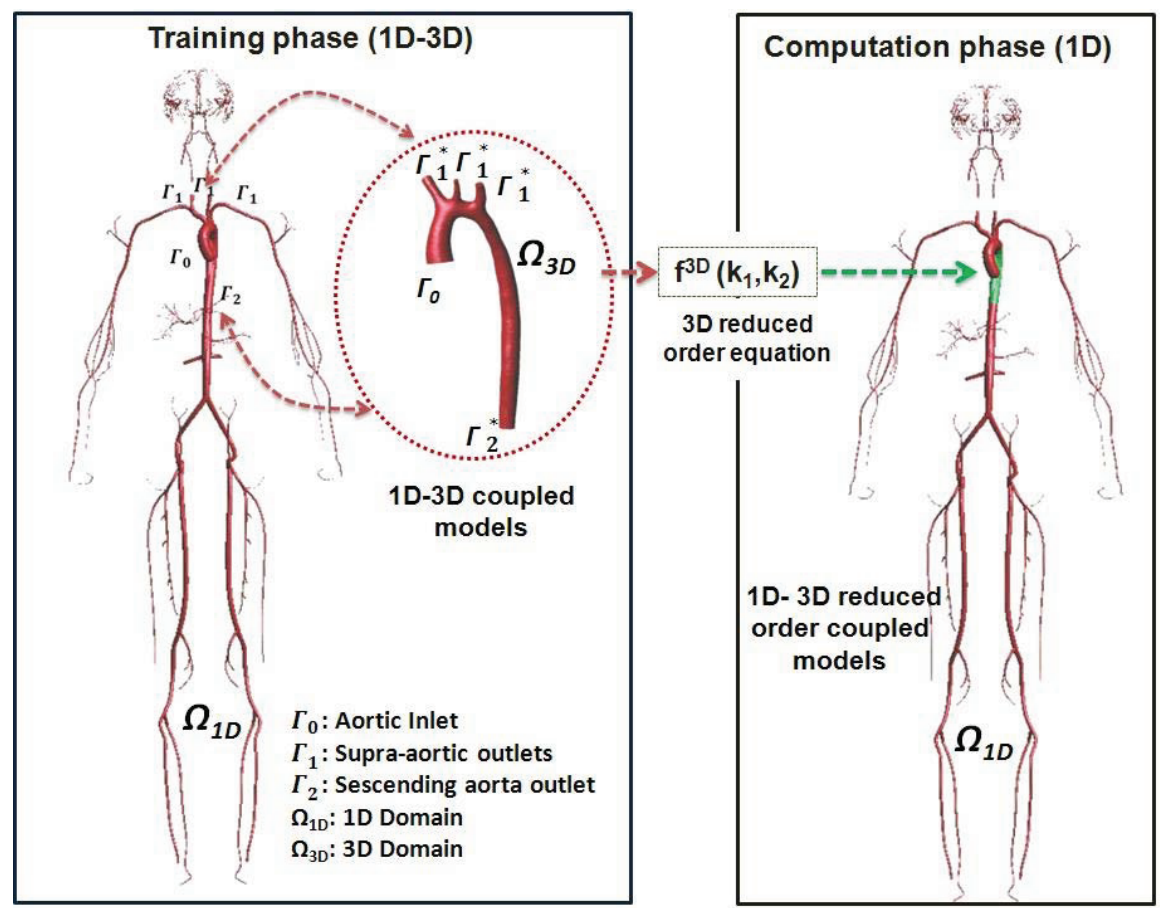

Fig. $13 \mathrm{D}-1 \mathrm{D}$ coupled approach schematics. $\Gamma_{i}$ are the interface surfaces

$$
\begin{aligned}
\left(\frac{\partial \mathbf{A}}{\partial \mathbf{t}}+\frac{\partial \mathbf{Q}}{\partial \mathbf{z}}\right) & =0 \\
\left(\frac{\partial \mathbf{Q}}{\partial t}\right)+\frac{\partial}{\partial z}\left(\alpha \frac{Q^{2}}{A}\right)+\frac{\mathbf{A}}{\rho} \frac{\partial \mathrm{P}}{\partial z}+\mathbf{K}_{\mathbf{R}}\left(\frac{Q}{A}\right) & =0 \\
P_{\text {ext }}+\frac{E h_{o} \sqrt{\Pi}}{1-\mu^{2}} \frac{\sqrt{A}-\sqrt{A_{o}}}{A_{o}} & =P
\end{aligned}
$$

where $A(z, t)$ is the cross-sectional area of the vessel, $Q(z, t)$ is the mean blood flow, $\mathrm{P}$ is the average internal pressure over the cross-section, $\alpha$ is the momentumflux correction coefficient, $\mathrm{z}$ is the axial coordinate along the vessel, $\mathrm{t}$ is the time, $\rho$ is the density of the blood taken as $1050 \mathrm{Kg} / \mathrm{m}^{3}$ and $\mathrm{K}_{R}$ is the friction force per unit length, which is modeled as $\mathrm{K}_{R}=2 \pi \cdot v(\gamma+2)$ [17], with $v$ being the viscosity of the blood taken here as $4.5 \mathrm{~m} \cdot \mathrm{Pa} \cdot \mathrm{s}$. The vessel wall is modeled as a thin, homogeneous and elastic material. Parameters $\mathrm{A}_{0}$ and $\mathrm{h}_{0}$ in Eq.1(c) are the sectional area and the wall thickness, respectively, at the reference state $\left(\mathrm{P}_{0}, \mathrm{U}_{0}\right)$, with $\mathrm{P}_{0}$ and $\mathrm{U}_{0}$ assumed to be zero, $\mathrm{E}$ is the Young modulus and $\mu$ is the Poisson's ratio, typically taken as $\mu \approx 0.5$, which implies that the biological tissue is practically incompressible. In absence of detailed of patient-specific data, the wall elasticity and the thickness of the 55 largest arteries are based on data published by Wang and Parker [12]. At each 
domain decomposition, whether corresponding to a discontinuity in the mechanical or geometrical vessel properties, or to a cardiovascular branching, continuity of flow and of total pressure is enforced as follows:

$$
\begin{gathered}
Q_{i}=\sum_{d}\left(Q_{j}\right)_{d} \\
P_{i}+\frac{1}{2} \rho \frac{Q_{i}^{2}}{A_{i}^{2}}=\left(P_{j}\right)_{d}+\frac{1}{2} \rho \frac{\left(Q_{j}^{2}\right)_{d}}{\left(A_{j}^{2}\right)_{d}}+\left(f^{3 D}{ }_{j}\left(k_{1}, k_{2}\right)\right)_{d}
\end{gathered}
$$

where indexes $i$ and $j$ denote the parent and the daughter vessels respectively, and $d$ indicates the number of system domains. Function $\mathrm{f}^{3 D}\left(\mathrm{k}_{1}, \mathrm{k}_{2}\right)$ denotes the energy losses of the 3D model, where $\mathrm{k}_{1}$ and $\mathrm{k}_{2}$ are obtained by fitting the pressure drop between the two planes defined in the $3 \mathrm{D}$ model. $\mathrm{k}_{1}$ and $\mathrm{k}_{2}$ are the viscous and turbulent coefficients that should be adjusted according to the pressure drop between the two planes defined in the 3D model. In this work we do not consider the inertial term. The system obtained is solved by a Newton iteration scheme, taking as the starting point the reference section area and flow, i.e:

$$
f^{3 D}{ }_{j}\left(k_{1}, k_{2}\right)=k_{1} Q_{j}+k_{2}\left|Q_{j}\right| Q_{j}
$$

With simple manipulations of the differential equations (1) it is possible to obtain the conservative form for the temporal evolution of the flow and the vessels area and discretize the system obtained using a second order Taylor-Galerkin scheme. This scheme is appropriate for this problem as it can propagate waves of different frequencies without suffering from excessive dispersion and diffusion errors. A derivation of the 1D-FSI models can be found in [17] and [32]. The Taylor-Galerkin scheme requires a time step limitation in order to keep the solution stable. In this work the stabilization technique adopted has been the Courant Friedrichs Lewy condition (CFL condition)[21].

$$
\Delta t \leq C F L \min _{0 \leq i \leq N}\left(\frac{h_{i}}{\max \left(\lambda_{1, i}, \lambda_{1, i+1}\right)}\right)
$$

where $N$ is the number of the elements, $h_{i}$ is the local element size and $\lambda_{1, i}$ indicates the value of the eigenvalue evaluated at the mesh $\mathrm{i}^{\text {th }}$ node of the matrix of the conservative form obtained from the derivation of the 1D-FSI model[17]. The CFL value adopted is $0.57[21]$. The $3 \mathrm{D}$ computational analysis is performed assuming that the arterial wall is rigid. Blood is considered as an homogeneous laminar Newtonian fluid modelled by the incompressible Navier-Stokes equations using the same density and dynamic viscosity as for the 1D model. Although these are important limitations, they make the simulation effort simpler. Furthermore, the knowledge of the patient-specific mechanical properties is quite difficult, consequently the objective of this work is to determine the pressure gradient in the anatomical domain using a reduced-order model based on multiscale modeling. Recent studies [9] use turbulence models to predict the kinetic energy due to the narrowing of the coarctation. 


\subsection{D-1D Coupling interfaces}

In order to keep the continuity in the area sections between the 3D and the $1 \mathrm{D}$ geometrical models, the diameters of the 1D geometrical model were firstly scaled taking into account a proportional diameter factor between the 3D and 1D geometrical models. The properties of the 1D geometrical models were taken from [12]. For the training phase, at each coupling 1D-3D interface we enforce the continuity of the flow and the total pressure (ecs.2,3). This means that at every time step $t_{n}$ we compute the velocity and the pressure using the 1D approach over the whole domain $\Omega_{1 D}$. Then, the variables over the interface sections $\left(\Gamma_{1}, \Gamma_{2}\right)$ of the $\Omega_{1 D^{-}} \Omega_{3 D}$ domain are determined (Figure 1). Following that, the 3D problem is solved in $\Omega_{3 D}$ using the boundary conditions obtained in the $\Gamma_{1}, \Gamma_{2}$ sections from the 1D model. For the next time step $\left(t_{n}+1\right)$ the process is repeated until the final simulation time is reached. This coupling procedure is justified by the fact that the $1 \mathrm{D}$ domain can be considered as a passive element which absorbs the flow generated by the 3-D domain. During the training phase, pressure values at $\Gamma_{1}$ and $\Gamma_{2}$ interfaces are stored for each time step with the objective of estimating the coefficients $\mathrm{k}_{1}$ and $\mathrm{k}_{2}$ of Ec.4 by the least squares method. We choose the value of $\mathrm{f}^{3 D}\left(\mathrm{k}_{1}, \mathrm{k}_{2}\right)$ that minimizes the sum of the squared pressure drop from the 1D flow values compared to the 3D values. In Section 3.1.3 we show a pressure drop of the 3D computational values versus the predictions of the reduced-order model (Fig.3). For the computation phase, the coupled 1D coupled FSI - reduced-order model is solved by using the coefficients $\mathrm{k}_{1}$ and $\mathrm{k}_{2}$ estimated previously.

\section{Study Case: Aorta Coarctation}

\subsection{Training phase}

\subsubsection{Model Anatomy, Geometry and mesh}

The physiological and geometrical data used in this work was obtained from [4].The patient was a $71 \mathrm{~kg}, 177 \mathrm{~cm}$ tall, 17 -year old male with a mild thoracic aortic coarctation. Image data come from a 1.5-T Phillips scanner using a gadolinium-enhanced MR angiography (MRA) with the patient in the supine position. The 3D model (Figure 2) includes the ascending aorta, aortic arch, descending aorta, left subclavii, brachiocephalic and finally left common carotid arteries in Stereo Lithography(STL) file format. To generate the 3D volume we used the pre and post-processor GiD [6]. GiD can be efficiently used for mesh generation in CFD analysis. For generating the 3D volume mesh from the STL file, we have used an isosurface stuffing procedure. This algorithm generates tetrahedral element form a small set of precomputed stencils. The boundary mesh is guaranteed to be a geometrically and topologically accurate approximation of the isosurface[35]. This technique ensures tetrahedral 
volume elements with optimal angle and volume for the simulations. Using this method we have obtained smooth elements and an aspect radio for the whole mesh greater than 0.9 (the ideal ratio is one for an equilateral triangle). In this work we dont need a refined boundary layer mesh, due to the we have focused on the energy dissipation induced by the turbulence effects. In [22] other authors propose how to build a patient-specific models from medical images taking into account a fine discretization of the boundary layer in order to capture the WSS and OSI effects over the arterial wall. The result of the isostuffing procedure is a volume mesh of 4,322,556 four-noded tetrahedral elements and 206,880 three-noded triangles with 777,235 nodes. The original surface mesh has 138,532 linear triangles and 69,268 nodes. Figure 2 shows a rendering image of the 3D volume mesh. The $1 \mathrm{D}$ computational mesh contains 551 linear two-noded elements and 586 nodes.

\subsubsection{Boundary conditions: Inlet and Outlet Conditions at Rest}

Blood flow information[4] was acquired using a cardiac-gated, 2D, respiratory compensated, phase-contrast (PC) sequence with through-plane velocity encoding. The cardiac output of the patient was $3.71 \mathrm{~L} / \mathrm{min}$, the heart rate was 47 beats per minute (cardiac cycle $\mathrm{T}=1.277 \mathrm{sec}$ ). Figure 2 shows the blood flow and pressure waveforms at the ascending aorta (AscAo). The inlet velocity profile is prescribed as uniform and flat at the inlet surface. The quality of the waveforms to the supra-aortic vessels (the Brachiocephalic artery(BA), the left common carotid artery(LCC) and the left subclavian artery(LS) arteries) was deemed too noisy to be used in the computations. Figure 2 and the related table show the total flow through each branch given as a percentage of the ascending aortic flow.

Invasive pressure wire measurements were acquired in a catheterization laboratoryequipped XMR suite [4]. Pressure was obtained in the ascending aorta (proximal to the coarctation). The proximal systolic, diastolic, and mean pressures were 83.92, 49.68, and $63.35 \mathrm{mmHg}$, respectively. These values were used to set the parameters for the lumped model. These lumped models are usually composed of a set of resistances and compliances to model the microvascular beds. The compliance influences the transient flow waveform, while the mean value is affected by the resistance only. Due to the assumption that only the mean flow over the cardiac cycle in the BA, LCC , LS and DiaphAo arteries is known, the boundary condition estimation is limited to correctly determining the resistance values at each of these outlets. For estimating the terminal resistance parameter, we use Ohm's law taking into account the flow distribution at the rest state(see the Table in Figure 2);

$$
Q_{i} * R_{i}=P_{i}-P_{\text {out }}
$$

where $\mathrm{Q}_{i}$ is the flow at the BA, LCC, LS and DiaphAo arteries, $\mathrm{P}_{i}$ is the mean systolic pressure at the aortic root, $\mathrm{P}_{\text {out }}$ is the venous pressure for the cardiovascular system and $\mathrm{R}_{i}$ is the flow resistance for each branch-domain. Since the circulation system is not closed, a constant venous pressure of $5 \mathrm{mmHg}$ is prescribed at the out- 


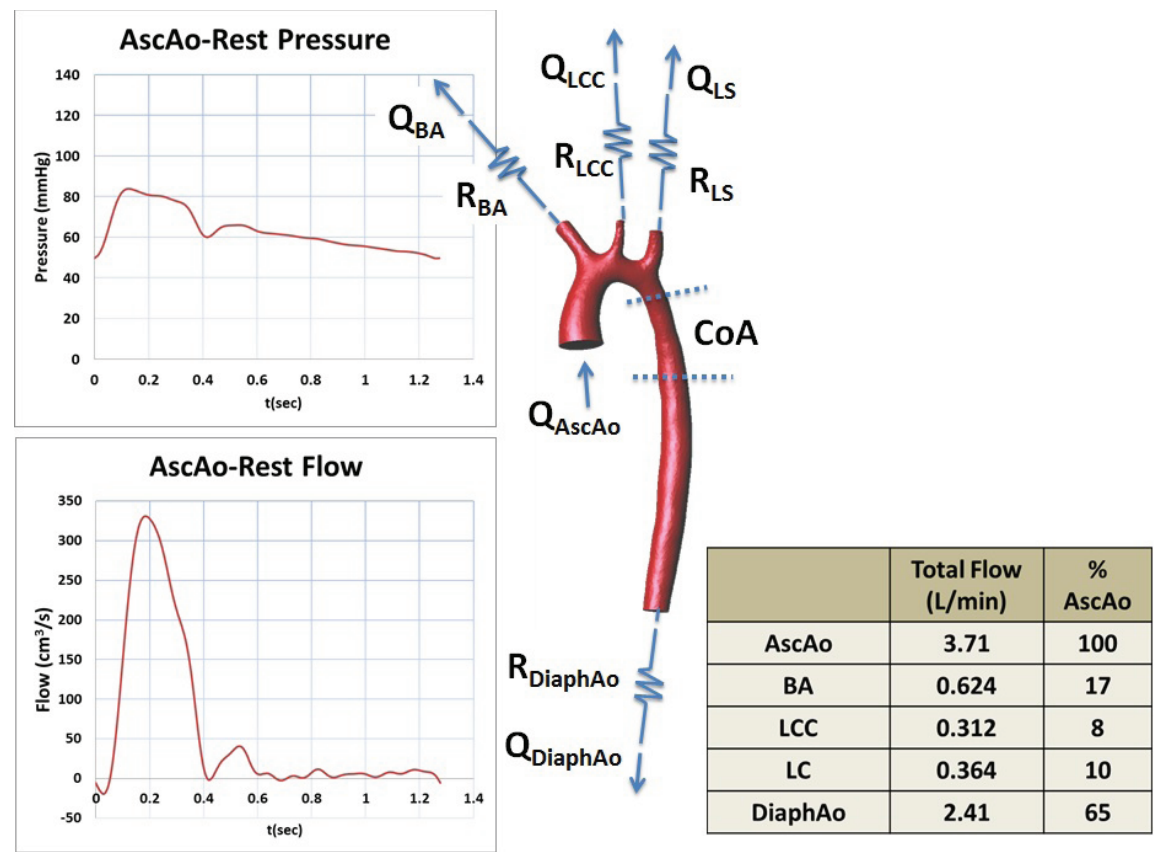

Fig. 2 Patient-specific anatomical model with a mild thoracic aortic coarctation (CoA). Ascending aortic flow waveforms (in $\mathrm{cm}^{3} / \mathrm{sec}$ ) under rest conditions measured by the (PC)-MRI sequence and ascending aortic pressure (in $\mathrm{mmHg}$ ) measured with a pressure catheter under rest conditions. The table shows the total flow (in $\mathrm{L} / \mathrm{min}$ ) and percentage of ascending aortic flow through the various branches of the aortic model under rest conditions

put of each artery[28]. Solving the set of differential-algebraic equations we obtain the following flow resistance for each branch as: $R_{B A}=7.48 \cdot 10^{11} \mathrm{~Pa} \cdot \mathrm{s} / \mathrm{m}^{3}, R_{L C C}=$ $1.50 \cdot 10^{12} \mathrm{~Pa} \cdot \mathrm{s} / \mathrm{m}^{3}, R_{L S}=1.28 \cdot 10^{12} \mathrm{~Pa} \cdot \mathrm{s} / \mathrm{m}^{3}$ and $R_{\text {DiaphAo }}=1.94 \cdot 10^{11} \mathrm{~Pa} \cdot \mathrm{s} / \mathrm{m}^{3}$. Once the total resistances are estimated at these branches, the next step is to distribute the total resistances to the lumped models for all outlets of the 1D cardiovascular domain. To do that we have used a technique based on scaling laws for estimating the blood resistance. This method assumes that the radii and the lengths can be approximated as $R_{1}=\varphi R_{0}$ and $L_{1}=\lambda L_{0}$, being $R_{0}$ and $L_{0}$ is the radii and the length of the artery at a further distance and $\varphi$ and $\lambda$ are constant scaling factors. Therefore for any artery the radii and the length are $R_{n}=\varphi R_{n-1}$ and $L_{n}=\lambda L_{n-1}$, respectively. Under the Poiseuille law and assuming that we have $2^{n}$ arteries, then the flow distribution is $Q_{0} / 2^{n}$, and the resistances in the terminal branches can be estimated as follows: 


$$
\begin{gathered}
\Delta p_{n}=Q_{n} \frac{8 L_{n} \mu}{\pi R_{n}^{4}} \Rightarrow \Delta p_{n}=\left\{\frac{\lambda}{2 \varphi^{4}}\right\}^{n} Q_{0} \frac{8 L_{0} \mu}{\pi R_{0}^{4}}=\left\{\frac{\lambda}{2 \varphi^{4}}\right\}^{n} \Delta p_{0} \\
\Delta p_{T}=\sum_{n=1}^{N} \Delta p_{n}=\Delta p_{0} \sum_{n=1}^{N}\left\{\frac{\lambda}{2 \varphi^{4}}\right\}^{n}
\end{gathered}
$$

where $\Delta p_{T}$ is the sum of the pressure gradients after each bifurcation through the network, $\Delta p_{n}$ is the starting point from the outlet of the terminal artery and $\mathrm{N}$ is the number of bifurcations. Dividing $\Delta p_{T}$ by $Q_{0}$, the resistance $R_{\mu} T$ can be estimated as:

$$
R_{\mu T}=R_{\mu 0} \sum_{n=1}^{N}\left\{\frac{\lambda}{2 \varphi^{4}}\right\}^{n}
$$

The flow can be approximated using Murray's law [5] as $Q \simeq k r^{3}$. Therefore $\lambda=\sqrt{0.6}$. Murray's law determines the vessel radius that requires a minimum of energy the vascular system. A relation between $\varphi$ and $\lambda$ can be estimated as [33]:

$$
\lambda=\frac{N}{N+1} \frac{1}{2 \varphi^{2}}
$$

On the other hand, considering the main 55 arteries of the 1D arterial model[12], the $R_{B A}, R_{L C C}, R_{L S}$ and $R_{\text {DiaphAo }}$ parameters and taking in account the vessels that arise from BA, LCC, LS and DiaphAo arteries the resistance values for all the terminal branches at the rest condition are finally estimated (see Table 1).

\subsubsection{Reduced-order model}

In order to settle eq.4, we have to define two planes in the 3D geometrical model. The planes chosen were in the ascending aorta (proximal to the coarctation) and in the descending aorta (distal to the coarctation); see Figure 2. Once these two planes and the inlet and boundary conditions for rest situations are defined, we solve the coupled 3D-1D model. We use an adaptative time step based on the 1D simulation . For each time step, we calculate and store the mean values of the pressure and blood flow for the two planes. These values are then used to estimate the $\mathrm{k}_{1}$ and $\mathrm{k}_{2}$ coefficients in (Ec.4) using a least squares method. The coefficients values found are $\mathrm{k}_{1}=3.08 \cdot 10^{-3}$ and $\mathrm{k}_{2}=5 \cdot 10^{-4}$, so that function $\mathrm{f}^{3 D}\left(\mathrm{k}_{1}, \mathrm{k}_{2}\right)$ minimizes the sum of the squared pressure drop from the flow values versus the $3 \mathrm{D}$ values. Figure 3(left) shows the pressure drop obtained with the $3 \mathrm{D}$ computational values and the reducedorder model. The mean error between the CFD pressure drop and the $3 \mathrm{D}$ reducedorder model is 3\%. Figure 3(right) shows the dependence between the pressure drop and the flow. The area below the hysteresis loop is the energy dissipated. If it is low we can estimate the pressure drop by function $\mathrm{f}^{3 D}\left(\mathrm{k}_{1}, \mathrm{k}_{2}\right)$. Thus, the reduced-order model is capable to capture the energy losses provoked by the geometry of the 3D model. The total computation time for the training phase in a standard PC with 
Linux environment, 32 bit, 4 GB RAM and dual core $2.83 \mathrm{GHz}$ CPU was about 10 hours.
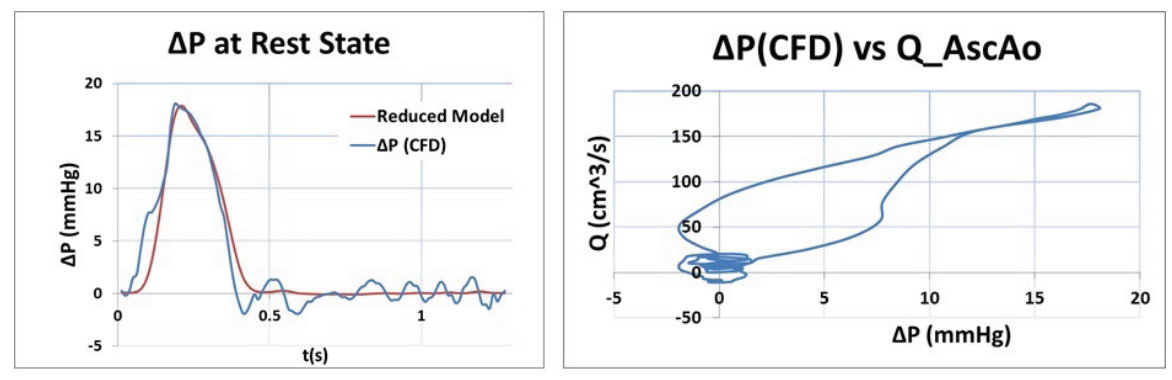

Fig. 3 Left:pressure drop between the 3D computational values versus the reduced-order model. Right: dependence between the pressure drop and the flow(hysteric loop)

\subsection{Computation phase}

\subsubsection{Non-rest situation}

Stress (or non-rest) conditions were induced by administering isoprenaline to the patient. Flow and pressure data waveforms were acquired using the same protocol as in rest conditions [4]. The cardiac output of the patient increased to $13.53 \mathrm{~L} / \mathrm{min}$ and the heart rate to 141 beats per minute (cardiac cycle $\mathrm{T}=0.425 \mathrm{sec}$ ). Figure 4 shows the flow waveform at AscAo. Similar as for a rest situation, the quality of the waveforms at the BA, LCC, and LS arteries was deemed too noisy to be used for the computations. Figure 4 shows the total flow through each branch given as a percentage of the ascending aortic flow. Pressure was obtained in the ascending aorta (proximal to the coarctation). The proximal systolic, diastolic, and mean pressures were $123.35,36.77$, and $64.30 \mathrm{mmHg}$, respectively (Figure 4). For estimating the terminal resistance parameters under non-rest situations we have used the same procedure as in rest situations (3.1.2). The results are $R_{B A}=1.41 \cdot 10^{11} \mathrm{~Pa} \cdot \mathrm{s} / \mathrm{m}^{3}, R_{L C C}=$ $6.90 \cdot 10^{11} \mathrm{~Pa} \cdot \mathrm{s} / \mathrm{m}^{3}, R_{L S}=3.25 \cdot 10^{11} \mathrm{~Pa} \cdot \mathrm{s} / \mathrm{m}^{3}$ and $R_{\text {DiaphAo }}=5.91 \cdot 10^{10} \mathrm{~Pa} \cdot \mathrm{s} / \mathrm{m}^{3}$. Table 1 shows the terminal resistance values for the terminal vessels for rest and non-rest situations.

Once the terminal resistances for the non-rest condition are set and the parameters $\mathrm{k}_{1}$ and $\mathrm{k}_{2}$ of the 3D reduced-order model are fitted (see Section 3.1.3). We are able to estimate the pressure drop under non-rest conditions. For this purpose, we impose the flow profile (Fig.4) in the ascending aorta of the 1D model. In Figure 5 the pressure drop under non-rest conditions is shown. The table below shows the mean, systolic and diastolic pressure for the rest and non-rest situations obtained with the reduced-order model. Experimental results are also shown for comparison 


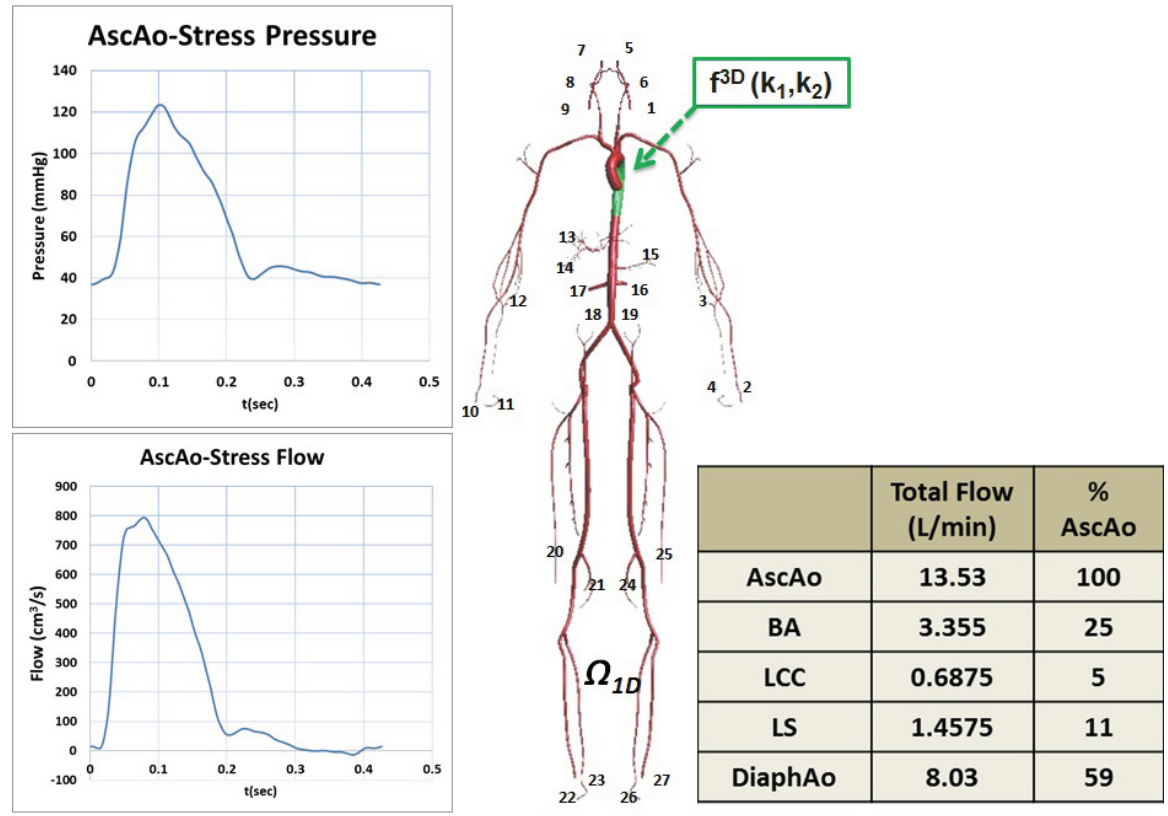

Fig. 4 1D anatomical model based on [12]. Ascending aortic flow waveforms (in $\mathrm{cm}^{3} / \mathrm{sec}$ ) under non-rest conditions as measured by the PC-MRI sequence and ascending aortic pressure (in $\mathrm{mmHg}$ ) measured with a pressure catheter under non-rest conditions. Table shows the total flow (in $\mathrm{L} / \mathrm{min}$ ) and percentage of ascending aortic flow through the various branches of the aortic model under non-rest conditions. Numbers indicate the terminal branches. Table 1 shows the name and resistance values.

Table 1 Resistance values for the cardiovascular model in Fig 4. R is the terminal resistance $\left(10^{9} \mathrm{~Pa}\right.$ $\mathrm{s} / \mathrm{m}^{3}$ ) for the rest and non-rest situations based on the experimental measurements from [12].

\begin{tabular}{lllllll}
\hline \multicolumn{1}{c}{ Artery name } & $\mathbf{R}_{\text {Rest }}$ & $\mathbf{R}_{\text {Non-rest }}$ & \multicolumn{1}{c}{ Artery name } & $\mathbf{R}_{\text {Rest }}$ & $\mathbf{R}_{\text {Non-rest }}$ \\
\hline 1 & Right Vertebral & 29.6 & 7.52 & 15 Intercostals & 4.47 & 1.36 \\
2 & Right radius & 0.68 & 0.14 & 16 Renal & 0.68 & 2.02 \\
3 & Right Interosseous & 4.5 & 1.14 & 17 Inferior Mesenteric & 0.68 & 2.02 \\
4 & Right Ulnar & 4.5 & 1.14 & 18 Superior Mesenteric & 0.68 & 2.02 \\
5 & Right int. carotid & 34.6 & 15.9 & 19 Splenic & 0.68 & 2.02 \\
6 & Right ext. carotid & 34.6 & 15.9 & 20 Left Ext. iliac & 0.10 & 0.0315 \\
7 & Left int. carotid & 29.6 & 7.52 & 21 Left Deep femoral & 0.10 & 0.0315 \\
8 & Left ext. carotid & 29.6 & 7.52 & 22 Left Posterior tibial & 0.0157 & 0.00479 \\
9 & Left Vertebral & 17.3 & 3.27 & 23 Left Anterior tibial & 0.0157 & 0.00479 \\
10 Left Radius & 0.4 & 0.075 & 24 Right Ext. iliac & 0.10 & 0.0315 \\
11 Left Interosseous & 2.63 & 0.49 & 25 Right Deep femoral & 0.0157 & 0.00479 \\
12 Left Ulnar & 2.63 & 0.49 & 26 Right Posterior tibial 0.0157 & 0.00479 \\
13 Hepatic Superior & 4.47 & 1.36 & 27 Right Anterior tibial & 0.10 & 0.0315 \\
14 Gastric & 4.47 & 1.36 & & & \\
\hline
\end{tabular}


purposes. The 1D reduced-order model can be also used to simulate other pathological conditions without the necessity to perform the 3D simulation.

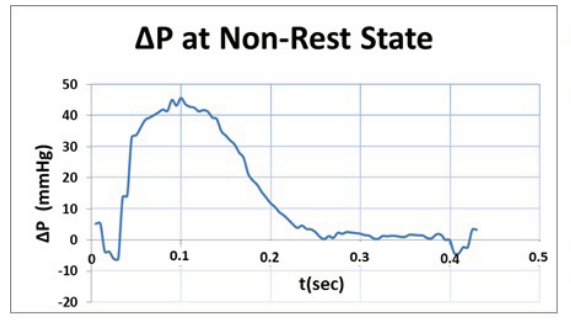

\begin{tabular}{|c|c|c|c|c|}
\hline & $\begin{array}{c}\text { Rest } \\
\text { (CFD) }\end{array}$ & $\begin{array}{c}\text { Rest } \\
\text { (Exp) }\end{array}$ & $\begin{array}{c}\text { Non-Rest } \\
\text { (CFD) }\end{array}$ & $\begin{array}{c}\text { Non-Rest } \\
\text { (Exp) }\end{array}$ \\
\hline Mean Pressure & 60.61 & 63.35 & 61 & 64.30 \\
\hline Max Pressure & 97.99 & 83.917 & 136.71 & 123.35 \\
\hline Min Pressure & 46.37 & 49.68 & 31.59 & 36.77 \\
\hline Mean $\triangle \mathrm{P}$ CoA & 2.83 & - & 13.86 & - \\
\hline Max $\triangle \mathrm{P}$ CoA & 18.07 & - & 45.66 & - \\
\hline
\end{tabular}

Fig. 5 Pressure drop for non-rest situation and CFD results versus experimental results. Pressures are in $\mathrm{mmHg}$. CoA represents the coarctation and $\Delta \mathrm{P}$ the pressure drop

\section{Results}

From a qualitative point of view, the numerical results shows that the pressure is correctly captured by the reduced-order model (fig.3). For the rest situation, the mean $(2.83 \mathrm{mmHg})$ and maximum $(18.07 \mathrm{mmHg})$ pressure drops are estimated using the 3D-1D coupling model. For the non-rest situation, the mean and maximum pressure drops of 13.68 and $44.06 \mathrm{mmHg}$ are estimated using the 1D-reduced-order model. These values are in agreement with previous studies [4][11]. In the rest situation, the reduced model is able to capture the pressure drop with an error under 3\%. Note that the methodology used for parameter estimation can be improved with the objective of avoiding the oscillatory behavior of the pressure in the descending aorta. Some authors [23][24] use the three-element Windkessel model for improving the fitting of the lumped model. The approach used in this work is however much simpler in the mathematical treatment, while still being able to match the systolic, diastolic and mean pressure values.

\section{Conclusion}

We have presented a CFD framework which combines patient-specific model and a reduced-order model to estimate the energy losses in cardiovascular problems. The framework has been evaluated with a patient-specific aortic coarctation $(\mathrm{CoA})$ under non-rest and rest situations with satisfactory results. The procedure consists of a two step process. First a reduced-order model is trained using a coupled 1D-3D FEM analysis and then is used together with a 1D solver under different pathological conditions. The reduced-order model is expressed as a sum of the viscous and turbulent 
terms, and it is capable to capture the energy losses provoked by the anatomy shape. The error of the reduced-order model is acceptable to capture the pressure drop over two sections of the 3D FEM model. We have modified the 1D formulation in order to integrate the reduced-order model into the framework developed. Results demonstrate that the reduced model is robust with respect to changes in the pathological conditions for patient-specific cases. In order to apply the procedure for a new patient, the framework developed requires a patient-specific model and knowledge of the pathological conditions in a rest situation. In terms of clinical diagnosis the most important feature to be captured is the pressure drop which can be estimated under non-rest situations. The main advantage of the proposed framework is that it relies only on measurements acquired during a rest situation. Then, it can be used under non-rest or rest conditions in a small computation time. The most important numerical approximation introduced in this work is that the training of the reduced-order model is performed using the 3D patient-specific anatomical data considering the boundary conditions taken from the $1 \mathrm{D}$ model. The advantage is a great reduction of the computational time versus the fully coupled analysis. The numerical results show that the 1D model retrofitted by using the trained reduced model correctly captures the pressure gradient and the energy losses in cardiovascular diseases.

\section{Acknowledgements}

This research was supported by the HFLUIDS project of the National RTD Plan of the Spanish Ministry of Science and Innovation I+D BIA2010-15880 and by the ERC Advanced Grant projects "REALTIME"(AdG-2009325) and "SAFECON"(AdG267521). We also thank Dr.Pooyan Dadvand for his support on the implementation of the reduced-order model in KRATOS and Mr.Maurizio Bordone for his help during the fitting of the terminal resistances during training and computation phases.

\section{References}

1. A. Cristiano, I. Malossi, PJ. Blanco, P. Crosetto, S. Deparis, and A. Quarteroni. Implicit coupling of one-dimensional and three-dimensional blood flow models with compliant vessels. Multiscale Modeling Simulation 11:2, 474-506, 2013.

2. A. Malossi, PJ. Blanco, P. Crosetto, S. Deparis and A. Quarteroni. Implicit Coupling of OneDimensional and Three-Dimensional Blood Flow Models with Compliant Vessels Multiscale Modeling Simulation 11:2, 474-506, 2013.

3. B.N. Steele, D. Valdez-Jasso, M.A. Haider and M.S. Olufsen. Predicting Arterial Flow and Pressure Dynamics Using a 1D Fluid Dynamics Model with a Viscoelastic Wall SIAM J. Appl. Math., 71(4), 1123-1143, 2011.

4. CFD Challange: Patient-Specific Hemodynamics at Rest and Stress through an Aortic Coarctation. 2013. http://www.vascularmodel.org/miccai2013/

5. C.D Murray. The Physiological Principle of Minimum Work: I. The Vascular System and the Cost of Blood Volume. Proceedings of the National Academy of Sciences of the United States of America 12 (3): 207-214. 1926 
6. GiD. The personal pre and postprocessor, http://www.gidhome.com CIMNE (2011).

7. I.E. Vignon-Clementel, A.C. Figueroa, K.E. Jansen, and C.A. Taylor. Outflow boundary conditions for three-dimensional finite element modeling of blood flow and pressure in arteries. Computer Methods in Applied Mechanics and Engineering. Vol.195, No.29-32, p. 3776-3796, 2006.

8. J. Alastruey, A.W. Khir, K.S. Matthys, P.Segers, S.J. Sherwin, P. R. Verdonck, K. H. Parker, J. Peir. Pulse wave propagation in a model human arterial network: Assessment of 1-D viscoelastic simulations against in vitro measurements J Biomech. August 11; 44(12): 2250-2258. 2011

9. J. Lantz, T. Ebbers, J. Engvall and M. Karlsson. Numerical and experimental assessment of turbulent kinetic energy in an aortic coarctation. Journal of Biomechanics, (46), 11, 1851-1858. 2013.

10. J.J LaDisa, R.J. Dholakia, C.A. Figueroa, I. E. Vignon-Clementel, F.P.Chan, M.M Samyn, J.R. Cava , C.A. Taylor, J.A. Feinstein. Computational simulations demonstrate altered wall shear stress in aortic coarctation patients treated by resection with end-to-end anastomosis. Congenital heart disease 6, 432-443, 2011.

11. J.J LaDisa et al. Computational simulations for aortic coarctation: representative results from a sampling of patients. Journal of Biomechanical Engineering 133(9), 091008-091017, 2011.

12. J. Wang and K.H. Parker. Wave propagation in a model of the arterial circulation. J.Biomeh, 37; 457-470, 2004.

13. K. Ralovich, et al. Hemodynamic assessment of pre-and post-operative aortic coarctation from MRI. In: Ayache, N., Delingette, H., Golland, P., Mori, K. (eds.) MICCAI 2012, Part II. LNCS, vol. 7511, pp. 486-493. Springer, Heidelberg, 2012.

14. K. Takizawa, J. Christopher, T.E. Tezduyar, and S. Sathe. Space-time finite element computation of arterial fluid-structure interactions with patient-specific data. International Journal for Numerical Methods in Biomedical Engineering, 26, 101-116, 2010.

15. K. Takizawa, C. Moorman, S. Wright, J. Purdue, T. McPhail, P.R. Chen, J. Warren, and T.E. Tezduyar. Patient-specific arterial fluid-structure interaction modeling of cerebral aneurysms. International Journal for Numerical Methods in Fluids, 65, 308-323, 2011.

16. K. Takizawa, C. Moorman, S. Wright, J. Christopher, TE. Tezduyar. Wall shear stress calculations in space-time finite element computation of arterial fluid-structure interactions. Comput Mech. 46:31-41, 2011.

17. L. Formaggia, D. Lamponi and A. Quarteroni. One dimensional model for blood flow in arteries. Journal of Engineering mathematis, $47: 251-276,2003$.

18. L. Formaggia, JF. Gerbeau, F. Nobile, and A. Quarteroni, On the coupling of 3D and 1D Navier-Stokes equations for flow problems in compliant vessels. Comp. Meth. Appl. Mech. Engrg., 191, pp. 561-582. 2001

19. L. Formaggia, F. Nobile, A. Quarteroni, A. Veneziani. Multiscale modelling of the circulatory system: a preliminary analysis. Comput.Visual.Sci.2, 75-83. 1999

20. L. Itu, P. Sharma, K. Ralovich, V. Mihalef, R. Ionasec, A. Everett, R. Ringel, A. Kamen, D. Comaniciu. Non-invasive hemodynamic assessment of aortic coarctation: validation with in vivo measurements. Ann. Biomed. Eng. 41, 669-681. 2013.

21. L. Quartapelle. Numerical solution of the incompressible Navier-Stokes equations. Birkhauser Verlag, Basel, 1993.

22. M. Manguoglu, K. Takizawa, A.H. Sameh, and T.E. Tezduyar. Solution of linear systems in arterial fluid mechanics computations with boundary layer mesh refinement. Computational Mechanics, 46, 83-89, 2010.

23. M. Ismail , W.A. Wall, M.G. Gee. Adoint-Based Inverse Analysis of Windkessel Parameters for Patient-Specific. Vascular Models. J. of Comp. Physics 244, 113170. 2013.

24. N. Xiao, J.D. Humphrey, C.A. Figueroa. Multi-Scale Computational Model of ThreeDimensional Hemodynamics within a Deformable Full-Body Arterial Network. J Comput Phys, 244: 22-40. 2013.

25. P. Dadvand, R. Rossi, E. Oate. An object-oriented environment for developing finite element codes for multi-disciplinary applications. Archives of Computational Methods in Engineering, 17, 253-297, 2010. 
26. P. Dadvand, R. Rossi, M. Gil, X. Martorell, J. Cotela, E. Juanpere, S.R. Idelsohn, E. Oate. Migration of a generic multi-physics framework to HPC environments. Computers and fluids. Volume 80, 10, Pages 301-309. July 2013.

27. P. Perdikaris and G.E. Karniadakis. Fractional-Order Viscoelasticity in One-Dimensional Blood Flow Models. Annals of Biomedical Engineering, 0090-6964, 2014.

28. P. Reymond. Pressure and Flow Wave Propagation in Patient-Specific Models of the Arterial Tree. PhD Thesis, cole Polytechnique Fdrale de Lausanne, 2011.

29. P. Reymond, F. Merenda, F. Perren, D. Rafenacht, N. Stergiopulos. Validation of a onedimensional model of the systemic arterial tree. American Journal of Physiology-Heart and Circulatory Physiology, 297(1) H208-H222, 2009.

30. R. Raghu, I.E. Vignon-Clementel , C.A. Figueroa, C.A. Taylor. Comparative study of viscoelastic arterial wall models in nonlinear one-dimensional finite element simulations of blood flow. J Biomech Eng. 133, 081003-081011, 2011.

31. R. Torii, M. Oshima, T. Kobayashi, K. Takagi, TE. Tezduyar. Fluid structure interaction modeling of blood flow and cerebral aneurysm: significance of artery and aneurysm shapes. Comput Methods Appl Mech Eng 198:3613-3621, 2009.

32. S.J. Sherwin, V. Franke, J. Peir K.H. Parker. One-dimensional modelling of a vascular network in space-time variables. J. Engrg. Math. 47, 217-250, 2003.

33. T.F. Sherman. On connecting large vessels to small. The meaning of Murray's law. The Journal of General Physiology 78 (4): a 431-453. 1981.

34. T.E. Tezduyar, K. Takizawa, T. Brummer, and P.R. Chen. Space-time fluid-structure interaction modeling of patient-specific cerebral aneurysms. International Journal for Numerical Methods in Biomedical Engineering, 27, 665-1710, 2011.

35. W.E. Lorensen and H. E. Cline. Marching cubes: A high reso-lution 3d surface construction algorithm. In Proceedings of SIGGRAPH, pages 163-169, 1987.

36. Y. Bazilevs, K. Takizawa, T.E. Tezduyar. Computational Fluid-Structure Interaction: Methods and Applications. ISBN: 978-0-470-97877-1. 404 pages. 2013. 